\title{
Neutrino signature of supernova hydrodynamical instabilities in three dimensions
}

\author{
Irene Tamborra, ${ }^{1}$ Florian Hanke, ${ }^{2}$ Bernhard Müller, ${ }^{2}$ Hans-Thomas Janka, ${ }^{2}$ and Georg Raffelt ${ }^{1}$ \\ ${ }^{1}$ Max-Planck-Institut für Physik (Werner-Heisenberg-Institut), Föhringer Ring 6, 80805 München, Germany \\ ${ }^{2}$ Max-Planck-Institut für Astrophysik, Karl-Schwarzschild-Str. 1, 85748 Garching, Germany
}

(Dated: September 20, 2013)

\begin{abstract}
The first full-scale three-dimensional (3D) core-collapse supernova (SN) simulations with sophisticated neutrino transport show pronounced effects of the standing accretion shock instability (SASI) for two high-mass progenitors $\left(20\right.$ and $\left.27 M_{\odot}\right)$. In a low-mass progenitor $\left(11.2 M_{\odot}\right)$, large-scale convection is the dominant nonradial hydrodynamic instability in the postshock accretion layer. The SASI-associated modulation of the neutrino signal $(80 \mathrm{~Hz}$ in our two examples) will be clearly detectable in IceCube or the future Hyper-Kamiokande detector, depending on progenitor properties, distance, and observer location relative to the main SASI sloshing direction. The neutrino signal from the next galactic SN can, therefore, diagnose the nature of the hydrodynamic instability.
\end{abstract}

PACS numbers: 97.60.Bw, 14.60.Lm

Introduction.-Bethe and Wilson's delayed neutrinodriven explosion mechanism [1] remains the standard core-collapse SN paradigm [2]. At core bounce a shock wave forms, stalls after reaching $100-200 \mathrm{~km}$, and is revived by neutrino heating after tens to hundreds of ms, depending on progenitor properties and accretion rate of stellar matter that continues to collapse. One modern key ingredient to this scenario is its inherent multidimensional nature inferred from observed SN asymmetries [3] and from parametric and self-consistent 2D and 3D hydrodynamical simulations [4 7]. During the accretion phase, large-scale convective overturn develops in the neutrino-heated postshock layer [8] and the standing accretion shock instability (SASI) can arise, involving global dipolar and quadrupolar deformation and sloshing motions of the shock front [9, 10] as well as spiral modes 11 15. The next galactic SN may reveal these effects in gravitational waves 16 19 and in neutrino flux variations [20, 21].

Most SN investigations of convection and SASI have relied on axisymmetric simulations where sloshing motions are constrained to the symmetry axis [16, 22 27]. Several recent 3D models have treated neutrino heating and cooling in the SN core in various approximations 28 34]. They found SASI sloshing motions with considerably reduced amplitudes and stochastically changing direction or no clear SASI signature at all. Buoyancydriven convection was concluded to dominate post-shock turbulence and SASI to be a minor feature of SN dynamics at best 34 36. However, self-consistent, 2D, general relativistic simulations with sophisticated neutrino transport suggest that a genuine SASI remains possible if the shock stagnation radius is sufficiently small [25]. SASI development may depend on both, progenitor properties and the exact behavior of the stalled shock, which requires reliable neutrino transport. So the importance of the SASI relative to neutrino-driven convection remains controversial. Therefore it is remarkable that the first $3 \mathrm{D}$ simulation with detailed neutrino transport (a $27 M_{\odot}$ model) shows violent SASI activity [15].

SASI activity strongly modulates the accretion flow to the neutron star and the associated neutrino emission 16, 22]. The detection of such fast time variations of the neutrino signal will offer a unique chance to probe stellar core collapse and its detailed astrophysics 20, 21]. A significant signal must stick above the shot noise caused by the fluctuating event rate. IceCube [37, 38] is among the most promising facilities for this task, detecting a large number of Cherenkov photons triggered by neutrinos. Moreover, Super-Kamiokande (Super-K) 39], or the next-generation Hyper-Kamiokande (Hyper-K) [40], although with smaller rate than IceCube, will monitor the neutrino signal without background and will provide event-by-event energy information. The once-in-alifetime opportunity to observe a high-statistics SN neutrino signal provides one of several physics motivations to build, maintain, and constantly improve such large neutrino observatories.

We here study the detection opportunities for a SASImodulated SN neutrino signal based on the world-wide first 3D simulations with detailed neutrino transport of three progenitors with $27 M_{\odot}$ [15], $20 M_{\odot}$, and $11.2 M_{\odot}$. Whenever vigorous SASI motions grow despite neutrinodriven convection, the neutrino signal modulations will be clearly detectable for a galactic SN, but the exact signal features depend on progenitor properties, SN distance, and location of the observer relative to the main sloshing directions.

Numerical supernova models.-We use solar metallicity progenitors for which the evolution until the onset of iron-core collapse has been reported in [41] for the 11.2 and $27 M_{\odot}$ stars and in [42] for the $20 M_{\odot}$ star. They were previously employed for $2 \mathrm{D}$ simulations 23225,43$]$. Our 3D modeling uses the Prometheus-Vertex hydrodynamics code. It includes a "ray-by-ray-plus" (RbR+), fully velocity and energy-dependent neutrino transport module based on a variable Eddington-factor technique that solves iteratively the neutrino energy, momentum, 
and Boltzmann equations [44, 45]. We employ state-ofthe-art neutrino interaction rates 24,45$]$ and relativistic gravity and redshift corrections [44, 46].

The RbR+ description assumes the neutrino momentum distribution to be axisymmetric around the radial direction everywhere, implying that the neutrino fluxes are radial. The detectable energy-dependent neutrino emission from the hemisphere facing an observer is determined with a post-processing procedure that includes projection and limb-darkening effects [30]. We will use the $27 M_{\odot}$ model as our benchmark case because its properties have been published [15]. Details of the other two simulations will be provided elsewhere [47]. All simulations used artificial random density perturbations of $0.1 \%$ amplitude on the whole numerical grid to seed the growth of hydrodynamic instabilities. None of the models had exploded at the end of the computation runs.

Detector signal.- In the largest operating detectors, IceCube and Super-K, neutrinos are primarily detected by inverse beta decay, $\bar{\nu}_{e}+p \rightarrow n+e^{+}$, through Cherenkov radiation of the positron. We represent the neutrino emission spectra in the form of Gamma distributions [48, 49]. We estimate the neutrino signal following the IceCube Collaboration [37], accounting for a 13\% deadtime effect for background reduction. We use a cross section that includes recoil effects and other corrections [50], overall reducing the detection rate by $30 \%$ relative to earlier studies [20, 21, 51]. On the other hand, we increase the rate by $6 \%$ to account for detection channels other than inverse beta decay [37].

We assume an average background of $0.286 \mathrm{~ms}^{-1}$ for each of the 5160 optical modules, i.e., an overall background rate of $R_{\mathrm{bkgd}}=1.48 \times 10^{3} \mathrm{~ms}^{-1}$, comparable to the signal rate for a SN at $10 \mathrm{kpc}$. The IceCube data acquisition system has been upgraded since the publication of Ref. [37] so that the full neutrino time sequence will be available instead of time bins.

IceCube will register in total around $10^{6}$ events above background for a SN at $10 \mathrm{kpc}$, to be compared with around $10^{4}$ events for Super-K (fiducial mass $32 \mathrm{kton}$ ), i.e., IceCube has superior statistics. On the other hand, the future Hyper-K will have a fiducial mass of $740 \mathrm{kton}$, providing a background-free signal of roughly $1 / 3$ the IceCube rate. Therefore, Hyper-K can have superior signal statistics, depending on SN distance. In addition, it has event-by-event energy information which we do not use for our simple comparison.

Signal modulation in the $27 M_{\odot}$ model.-To get a first impression of the neutrino signal modulation we consider our published $27 M_{\odot}$ model [15], meanwhile simulated until $\sim 550 \mathrm{~ms}$. This model shows clear SASI activity at 120-260 ms. At $\sim 220 \mathrm{~ms}$ a SASI spiral mode sets in and remains largely confined to an almost stable plane, which is not aligned with the polar grid of the simulation. We select an observer in this plane in a favorable direction and show the expected IceCube signal in the top panel
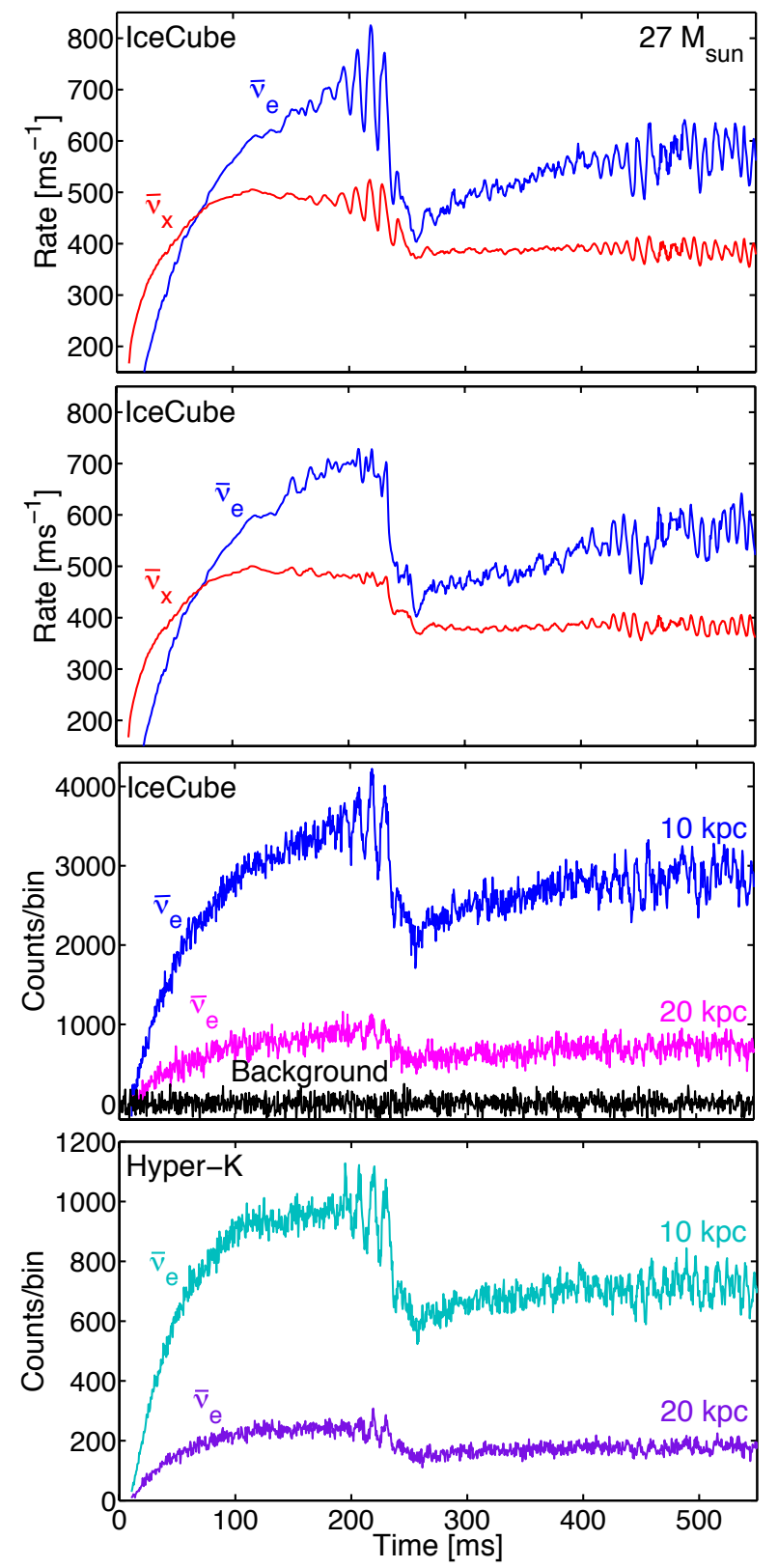

FIG. 1: Detection rate for our $27 M_{\odot}$ SN progenitor, upper panels for IceCube, bottom one for Hyper-K. The observer direction is chosen for strong signal modulation, except for the second panel (minimal modulation). Upper two panels: IceCube rate at $10 \mathrm{kpc}$ for $\bar{\nu}_{e}$ (no flavor conversion) and for $\bar{\nu}_{x}$ (complete flavor conversion). The lower two panels include a random shot-noise realization, $5 \mathrm{~ms}$ bins, for the indicated SN distances. For IceCube also the background fluctuations without a SN signal are shown.

of Fig. 1. One case assumes the signal to be caused by anti-neutrinos emitted as $\bar{\nu}_{e}$ at the source, i.e., we ignore flavor conversions. The other case takes into account complete flavor conversion so that the signal is caused by $\bar{\nu}_{x}$, i.e., a combination of $\bar{\nu}_{\mu}$ and $\bar{\nu}_{\tau}$. Both cases reveal large signal modulations with a clear periodicity. 


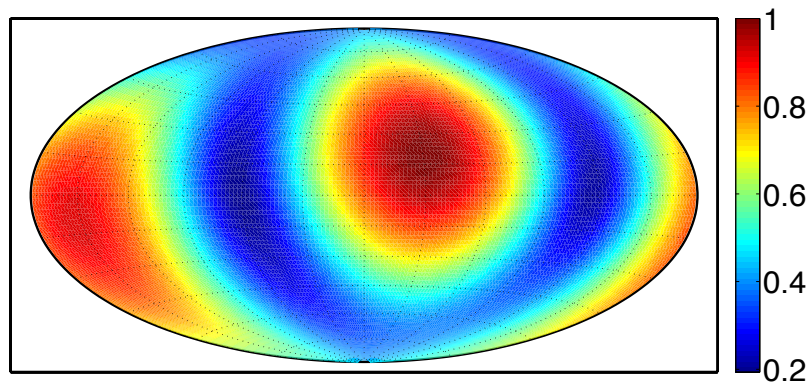

FIG. 2: Relative amplitude of the $\bar{\nu}_{e}$ rate modulation (see Eq. 1) on a sky-plot of observer directions during the first SASI episode $(120-250 \mathrm{~ms})$ of the $27 M_{\odot}$ model.

The first SASI episode ends abruptly with the accretion of the $\mathrm{Si} / \mathrm{SiO}$ interface, followed by large-scale convection with much smaller and less periodic signal modulations (see also Figs. 1, 2, and 6 of Ref. [15]). After about $410 \mathrm{~ms}$, SASI activity begins again until the end of our simulation. The signal modulation is now weaker, partly owing to a lower SASI amplitude and partly to the chosen observer direction being no longer optimal.

The second panel of Fig. 1 1 is for a direction orthogonal to the plane of the first SASI episode, i.e., the signal modulation is particularly small. The second SASI episode now shows a stronger signal than the first because the observer is no longer in the worst direction.

The SASI sloshing and spiral motions imply that observers in opposite directions obtain almost the same signal modulations with opposite phase. To illustrate the dependence on the observer direction we provide a supplementary movie on the time-evolution of the IceCube rate ${ }^{1}$. As a static visualization we show in Fig. 2 the relative amplitude of the IceCube detection rate during the first SASI episode. To define this amplitude we first note that the signal rate, averaged over all directions, hardly shows any modulation at all. In a given direction we define the relative time-dependent rate and consider its root mean square deviation for the first SASI episode $\left(\left[t_{1}, t_{2}\right]=[120,250] \mathrm{ms}\right)$,

$$
\sigma \equiv\left(\int_{t_{1}}^{t_{2}} d t\left[\frac{R-\langle R\rangle}{\langle R\rangle}\right]^{2}\right)^{1 / 2} .
$$

Despite the spiral mass motions during this SASI episode and the corresponding, considerable time variability of the emission asymmetry, the time integrated analysis still reveals a dominant sloshing direction, which produces two signal "hot spots" in two opposite directions, surrounded by directions with much smaller modulations.

\footnotetext{
1 http://www.mpa-garching.mpg.de/ccsnarchive/data/ Hanke2013_movie/index.html.
}

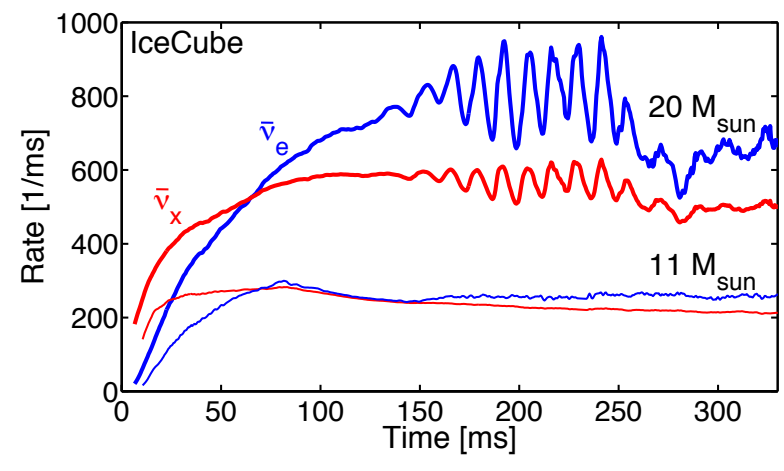

FIG. 3: IceCube rate for optimal observing directions for the 11.2 and $20 M_{\odot}$ models at $10 \mathrm{kpc}$, as in the top panel of Fig. 1.

Other progenitors.-Figure 3 shows the IceCube rate for the other progenitors (11.2 and $20 M_{\odot}$ ) in optimal observing directions. For the heavier case, a strong SASI develops after $140 \mathrm{~ms}$. Again a global SASI spiral mode largely confined to a plane appears, lasting until $\sim 300 \mathrm{~ms}$ close to the end of our simulation. The signal modulations are even more pronounced than for the $27 M_{\odot}$. progenitor and the SASI phase lasts slightly longer. In contrast, the $11.2 M_{\odot}$ model exhibits dominant activity by neutrino-driven convective overturn in the postshock layer (manifesting itself in a highly time-variable pattern of rising high-entropy bubbles and cooler downflows) without any clear signs of large-amplitude coherent SASI motions. In this case only very small, short-time signal fluctuations are visible for a chosen observer direction as a consequence of non-stationary, chaotically changing accretion anisotropies (similar to the cases analyzed in Refs. [21, 30]), although significant directional differences of the $\bar{\nu}_{e}$ signal can exist [47]. The detection rate is also much smaller because of a lower luminosity.

Shot noise.-The main limitation to observing signal modulations are random fluctuations in the detected neutrino time sequence. In the third panel of Fig. 1 we show the IceCube $\bar{\nu}_{e}$ signal in $5 \mathrm{~ms}$ bins, including a random shot noise realization. The signal is roughly $700 \mathrm{~ms}^{-1}$ near maximum, plus $1.48 \times 10^{3} \mathrm{~ms}^{-1}$ background, i.e., roughly $1.1 \times 10^{4}$ events per bin, causing a $\sim 3 \%$ random fluctuation of the signal itself where the average background is subtracted. We also show the IceCube signal in the absence of a SN, i.e., the background fluctuations alone. For a SN at $20 \mathrm{kpc}$, roughly the edge of the expected galactic SN distance distribution [54, 55], the signal is still visible to the naked eye, although the bin-to-bin fluctuation is now roughly $10 \%$.

In the bottom panel of Fig. 团 we show the analogous signal for Hyper-K, which has no background and thus yields roughly 900 events/bin. Its $3 \%$ bin-to-bin random fluctuation is almost identical to IceCube. Doubling the distance reduces the signal by four, but as there is no dark current, the fluctuations grow to about $7 \%$, i.e., at 


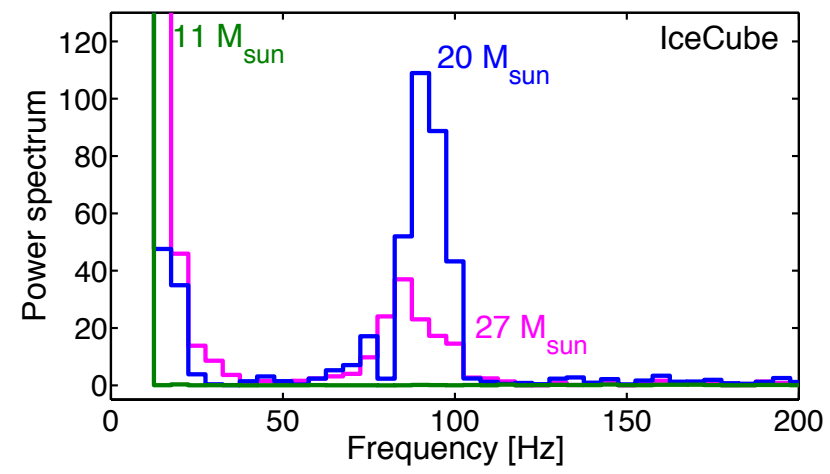

FIG. 4: Power spectrum of the IceCube event rate on the interval 100-300 ms for our three progenitors, assuming the $\bar{\nu}_{e}$ signal from a distance of $10 \mathrm{kpc}$. Normalization is to the frequency-independent power of shot noise caused by the IceCube background of $1.48 \times 10^{3} \mathrm{~ms}^{-1}$.

this distance Hyper-K is superior. We conclude that if the observer is located in an optimal direction, SASI can be detected throughout the galaxy.

A serious strategy to filter such signal modulations from the noise in less obvious cases is beyond the scope of our work. However, we also illustrate the signal in terms of its Fourier power spectrum, following Ref. 20]. We select the time interval of $100-300 \mathrm{~ms}$, where SASI develops for our progenitors. With the adopted signal duration of $\tau=200 \mathrm{~ms}$, the spacing of the discrete Fourier frequencies is $\delta f=1 / \tau=5 \mathrm{~Hz}$. We use a Hann window function on our interval to reduce edge effects in the Fourier transform. The minimum requirement for signal detection is that the Fourier spectrum sticks above background. The average power spectrum of a random signal sequence does not depend on frequency. Therefore, the IceCube dark current is a natural baseline and we use its power to normalize the signal power spectrum.

Figure 4 shows the power spectrum of the IceCube event rate for our three SN models thus normalized. A clear peak exists at $\sim 80 \mathrm{~Hz}$ for the two heavier progenitors where strong SASI appears. The modulation frequency is determined by the variations of the accretion flow that occur with the oscillation period of the SASI mode. The corresponding SASI (fundamental) frequency $f_{\mathrm{SASI}}$ depends roughly on the neutron star radius $R_{\mathrm{NS}}$ and shock radius $R_{\mathrm{S}}[10]$,

$$
f_{\mathrm{SASI}}^{-1} \sim \int_{R_{\mathrm{NS}}}^{R_{\mathrm{S}}} \frac{d r}{|v|}+\int_{R_{\mathrm{NS}}}^{R_{\mathrm{S}}} \frac{d r}{c_{\mathrm{S}}-|v|},
$$

where $c_{\mathrm{s}}$ and $v$ are the radius-dependent sound speed and accretion velocity, respectively, in the postshock layer. For both the 27 and $20 M_{\odot}$ progenitors the SASI frequencies are similar because of similar neutron star radii (the same equation of state is used) and mean shock radii in the first $250 \mathrm{~ms}$ after bounce.

Note that the amplitude of the signal power spectrum varies with the fourth power of distance. Therefore, doubling the distance to $20 \mathrm{kpc}$ reduces the power spectrum by a factor of 16 so that the main peak of the $27 M_{\odot}$ case reduces to about 3 times the shot-noise level.

Flavor oscillations.-Neutrinos change their flavor as they propagate from the emission region near the collapsed SN core to the detector. None of the mixing angles is especially small so that the propagation through the density gradient of the SN mantle and envelope leads to adiabatic Mikheev-Smirnov-Wolfenstein (MSW) conversions [52, 53]. In particular, this scenario predicts an approximately $70 \% \bar{\nu}_{e}$ survival probability in the normal hierarchy of neutrino masses, whereas in the inverted hierarchy, a complete swap $\bar{\nu}_{e} \leftrightarrow \bar{\nu}_{x}$ is expected.

This traditional picture may be strongly modified by collective flavor conversions between emission and the MSW region caused by neutrino-neutrino refraction. Our theoretical understanding of this effect is still developing. In the earlier literature one would have predicted an almost complete flavor swap in the anti-neutrino sector [56], reversing the hierarchy-dependence of the above MSW predictions. Then it was recognized that the ordinary matter effect can suppress collective flavor oscillations, especially during the accretion phase of high-mass progenitors where the matter density is large [57 60]. However, in those "high-density" cases the residual scattering of neutrinos beyond the emission region is strong enough to provide a "halo flux" with backward angular distribution, responsible for a significant modification of neutrino-neutrino refraction [61]. This effect most probably would not trigger collective flavor conversions in those cases where the matter effect is important [62]. The latest development is the presence of yet another instability due to the previously neglected azimuth variable of neutrino propagation, whose matter suppression would require larger densities than previously thought 63 65].

In view of these unresolved complications, it is not clear which exact flavor conversion scenario to expect as a function of the neutrino mass hierarchy and depending on the progenitor properties. For the purpose of detecting SASI-implied signal modulations, a complete flavor swap where we observe $\bar{\nu}_{e}$ that were born as $\bar{\nu}_{x}$ should be the worst case because of the smaller modulation amplitude, although still detectable.

Conclusions.- The first sophisticated 3D SN simulations show pronounced spiral SASI activity for the more massive of three different progenitors. There are SASI phases interspersed with episodes of dominant, largescale convective overturn activity. During the SASI periods, the neutrino signal modulations are even larger than those seen in previous 2D simulations, whereas the convective episodes are comparable to earlier $3 \mathrm{D}$ parametric cases [21, 30]. We have also shown that for SN distances beyond some $10 \mathrm{kpc}$, the future Hyper-K detector would be superior to IceCube. In spite of its smaller signal rate (about $1 / 3$ of IceCube), its lack of background implies a 
better signal-to-noise ratio because of reduced shot noise for those distances where IceCube is dominated by background fluctuations. To exploit the full Hyper-K potential, its event-by-event energy determination should be used as well.

The neutrino signal of the next galactic SN, if captured by IceCube and the future Hyper-K, offers a unique opportunity to diagnose different types of hydrodynamical instabilities. Such detectable instabilities appear in the first detailed 3D core-collapse SN simulations and depend on the progenitor properties.

Acknowledgments. - We thank Ewald Müller for discussions and Nicole Schwarz for the animated visualization. This research was partly supported by DFG through grants SFB/TR 7 and EXC 153 and by the EU under grant PITN-GA-2011-289442 (FP7 Initial Training Network "Invisibles"). I.T. acknowledges support by the Alexander von Humboldt Foundation. Our results were achieved with high-performance computing resources (Tier-0) provided by PRACE on CURIE TN (GENCI@CEA, France) and SuperMUC (GCS@LRZ, Germany), and with computing time on the IBM iDataPlex system hydra of the RZG.

[1] H. A. Bethe and J. R. Wilson, Astrophys. J. 295, 14 (1985).

[2] H.-T. Janka, Ann. Rev. Nucl. Part. Sci. 62, 407 (2012).

[3] W. D. Arnett, J. N. Bahcall, R. P. Kirshner and S. E. Woosley, Ann. Rev. Astron. Astrophys. 27, 629 (1989).

[4] M. Herant et al., Astrophys. J. 435, 339 (1994).

[5] A. Burrows, J. Hayes and B. A. Fryxell, Astrophys. J. 450, 830 (1995).

[6] H.-T. Janka and E. Müller, Astron. Astrophys. 306, 167 (1996).

[7] C. L. Fryer and M. S. Warren, Astrophys. J. Lett. 574, L65 (2002).

[8] H. A. Bethe, Rev. Mod. Phys. 62, 801 (1990).

[9] J. M. Blondin, A. Mezzacappa and C. DeMarino, Astrophys. J. 584, 971 (2003).

[10] L. Scheck, H.-T. Janka, T. Foglizzo and K. Kifonidis, Astron. Astrophys. 477, 931 (2008).

[11] J. M. Blondin and A. Mezzacappa, Nature (London) 445, 58 (2007).

[12] W. Iwakami et al., Astrophys. J. 700, 232 (2009).

[13] R. Fernández, Astrophys. J. 725, 1563 (2010).

[14] T. Foglizzo, F. Masset, J. Guilet and G. Durand, Phys. Rev. Lett. 108, 051103 (2012).

[15] F. Hanke et al., Astrophys. J. 770, 66 (2013).

[16] A. Marek, H.-T. Janka and E. Müller, Astron. Astrophys. 496, 66 (2009).

[17] K. Kotake, W. Iwakami, N. Ohnishi and S. Yamada, Astrophys. J. Lett. 697, L133 (2009).

[18] J. W. Murphy, C. D. Ott and A. Burrows, Astrophys. J. 707, 1173 (2009).

[19] B. Müller, H.-T. Janka and A. Marek, Astrophys. J. 766, 43 (2013).

[20] T. Lund et al., Phys. Rev. D 82, 063007 (2010).
[21] T. Lund et al., Phys. Rev. D 86, 105031 (2012).

$[22]$ T. D. Brandt, A. Burrows, C. D. Ott and E. Livne, Astrophys. J. 728, 8 (2011).

[23] A. Marek and H.-T. Janka, Astrophys. J. 694, 664 (2009).

[24] B. Müller, H.-T. Janka and A. Marek, Astrophys. J. 756, 84 (2012).

[25] B. Müller, H.-T. Janka and A. Heger, Astrophys. J. 761, 72 (2012).

[26] J. W. Murphy and A. Burrows, Astrophys. J. 688, 1159 (2008).

[27] J. Nordhaus, A. Burrows, A. Almgren and J. Bell, Astrophys. J. 720, 694 (2010).

[28] W. Iwakami et al., Astrophys. J. 678, 1207 (2008).

[29] A. Wongwathanarat, H.-T. Janka and E. Müller, Astrophys. J. 725, L106 (2010).

[30] E. Müller, H.-T. Janka and A. Wongwathanarat, Astron. Astrophys. 537, 63 (2012).

[31] F. Hanke, A. Marek, B. Müller and H.-T. Janka, Astrophys. J. 755, 138 (2012).

[32] T. Takiwaki, K. Kotake and Y. Suwa, Astrophys. J. 749, 98 (2012).

[33] C. D. Ott et al., Astrophys. J. 768, 115 (2013).

[34] A. Burrows, J. C. Dolence and J. W. Murphy, Astrophys. J. 759, 5 (2012).

[35] J. W. Murphy, J. C. Dolence and A. Burrows, Astrophys. J. 771, 52 (2013).

[36] J. C. Dolence, A. Burrows, J. W. Murphy and J. Nordhaus, Astrophys. J. 765, 110 (2013).

[37] R. Abbasi et al. (IceCube Collaboration), Astron. Astrophys. 535, A109 (2011).

[38] M. Salathe, M. Ribordy and L. Demirors, Astropart. Phys. 35, 485 (2012).

[39] K. Abe et al. (Super-Kamiokande Collaboration), Phys. Rev. D 83, 052010 (2011).

[40] K. Abe et al., arXiv:1109.3262.

[41] S. E. Woosley, A. Heger and T. A. Weaver, Rev. Mod. Physics 74, 1015 (2002).

[42] S. E. Woosley and A. Heger, Phys. Rep. 442, 269 (2007).

[43] S. W. Bruenn et al., Astrophys. J. Lett. 767, L6 (2013).

[44] M. Rampp and H.-T. Janka, Astron. Astrophys. 396, 361 (2002).

[45] R. Buras, M. Rampp, H.-T. Janka and K. Kifonidis, Astron. Astrophys. 447, 1049 (2006).

[46] A. Marek et al., Astron. Astrophys. 445, 273 (2006).

[47] I. Tamborra et al., in preparation.

[48] M. T. Keil, G. G. Raffelt and H.-T. Janka, Astrophys. J. 590, 971 (2003).

[49] I. Tamborra et al., Phys. Rev. D 86, 125031 (2012).

[50] A. Strumia and F. Vissani, Phys. Lett. B 564, 42 (2003).

[51] A. S. Dighe, M. T. Keil and G. G. Raffelt, JCAP 0306, 006 (2003).

[52] S. P. Mikheev and A. Yu. Smirnov, Sov. J. Nucl. Phys. 42, 913 (1985) [Yad. Fiz. 42, 1441 (1985)]; Sov. Phys. JETP 64, 4 (1986) [Zh. Eksp. Teor. Fiz. 91, 7 (1986)].

[53] A. S. Dighe and A. Yu. Smirnov, Phys. Rev. D 62, 033007 (2000).

[54] A. Mirizzi, G. G. Raffelt and P. D. Serpico, JCAP 0605, 012 (2006).

[55] S. M. Adams et al., arXiv:1306.0559.

[56] H. Duan, G. M. Fuller and Y.-Z. Qian, Ann. Rev. Nucl. Part. Sci. 60, 569 (2010).

[57] A. Esteban-Pretel et al., Phys. Rev. D 78, 085012 (2008).

[58] S. Chakraborty et al., Phys. Rev. Lett. 107, 151101 
(2011); Phys. Rev. D 84, 025002 (2011).

[59] S. Sarikas, G. G. Raffelt, L. Hüdepohl and H.-T. Janka, Phys. Rev. Lett. 108, 061101 (2012).

[60] N. Saviano, S. Chakraborty, T. Fischer and A. Mirizzi, Phys. Rev. D 85, 113002 (2012).

[61] J. F. Cherry et al., Phys. Rev. Lett. 108, 261104 (2012).

[62] S. Sarikas et al., Phys. Rev. D 85, 113007 (2012).
[63] G. G. Raffelt, S. Sarikas and D. de Sousa Seixas, Phys. Rev. Lett. 111, 091101 (2013).

[64] G. G. Raffelt and D. de Sousa Seixas, Phys. Rev. D 88, 045031 (2013)

[65] A. Mirizzi, arXiv:1308.1402. 